\title{
Percepção dos pais sobre os efeitos do treinamento auditivo acusticamente controlado em crianças
}

\author{
Perception of parents on the effects \\ of acoustically controlled hearing training \\ in children
}

\section{La percepción de los padres sobre los efectos del entrenamiento auditivo acústicamente controlado en niños}

\author{
Sandra Nunes Alves Viacelli* \\ Aline Bovolini* \\ Simone Sperança* \\ Sônia Faria* \\ Ricardo Oliveira Mello* \\ Pablo Felicio Nepomuceno* \\ Fátima Aparecida Gonçalves* \\ Liliane Desgualdo Pereira*
}

\begin{abstract}
* Universidade Federal de São Paulo - Unifesp, São Paulo, SP. Brasil
Este trabalho foi apresentado como pôster no XXIV Congresso Brasileiro de Fonoaudiologia, realizado de 20 a 23 de outubro de 2016 no Centro de Convenções Rebouças na cidade de São Paulo - SP.

Contribuição dos autores:

SNAV: Elaborou o projeto cientifico, realizou a intervenção direta junto aos participantes da pesquisa, coletou e participou da tabulação dos dados pré e pós-TAAC, participou da construção do texto científico deste manuscrito.

AB, PFN, FAG: Participaram da coleta de dados para a realização do trabalho.

SS: Contribuiu para a coleta de dados e tabulação dos mesmos nos momentos pré e pós-intervenção do treinamento auditivo acusticamente controlado, participou do levantamento bibliográfico, leitura e interpretação dos textos científicos que trouxeram embasamento teórico para esse manuscrito, participou ativamente da elaboração do texto, bem como, da construção dos resumos; colocou o texto, o quadro e as tabelas dentro das normas exigidas pela revista Distúrbios da Comunicação, bem como, realizou a submissão desse manuscrito à mesma.

SF: Contribuiu na tabulação dos dados de avaliação do PA e do TAAC pré e pós-intervenção; contribuiu na analise, na descrição e interpretação dos dados, participou ativamente da elaboração do texto, participou da definição de parâmetros e estratégias para construir o manuscrito. Foi responsável pela definição dos domínios, através da análise de cada questão da Tabela Sab, sob a ótica da psicologia comportamental.

ROM: Participou da tabulação dos dados para a realização do trabalho.

LDP: Orientou todos os integrantes do grupo e definiu os processos para a realização do trabalho cientifico; acompanhou, organizou e orientou todos os procedimentos realizados pelos membros do grupo desde a elaboração do projeto até a finalização do manuscrito.
\end{abstract}

E-mail para correspondência: Simone Sperança spmag4@hotmail.com

Recebido: $17 / 01 / 2017$

Aprovado: 25/07/2018 


\section{Resumo}

O uso de questionários pode auxiliar para verificar a percepção de pais/responsáveis sobre as habilidades auditivas dos filhos. Objetivo: Comparar a percepção dos pais de escolares submetidos ao treinamento auditivo acusticamente controlado, nos momentos inicial e final, por meio do questionário Scale Auditory Behavior, denominado "escala SAB". Método: 18 crianças ( $66,6 \%$ meninos), após terem sido diagnosticadas com Transtorno do Processamento Auditivo Centra (TPAC) com idades entre 6 e 13 anos (média de 10,4 anos) foram submetidas ao treinamento auditivo, que durou 15 sessões de 45 minutos cada. Todos os participantes tiveram melhora na avaliação comportamental pós-intervenção. Os responsáveis foram submetidos à escala $\mathrm{SAB}$ antes e depois do treinamento auditivo. O questionário contém 12 questões sobre o comportamento auditivo, cuja pontuação total varia de 12 (escore mais baixo) a 60 pontos (escore mais alto). As questões foram agrupadas por domínio: atenção, audição, aprendizado e compreensão auditiva. As respostas do questionário foram analisadas por domínio, segundo a pontuação ponderada analisada em valor absoluto, em porcentagem de dificuldade e evolução desse comportamento. Resultados: Na percepção dos responsáveis através da escala SAB, a maior melhora foi percebida no domínio da audição, seguida dos comportamentos auditivos de atenção, aprendizado e compreensão auditiva. Conclusão: Os pais das crianças submetidas à intervenção perceberam evolução do comportamento auditivo de seus filhos, com melhora satisfatória predominantemente nos domínios audição e atenção. Os domínios de compreensão e aprendizagem não atingiram a mesma melhora dos anteriores. A avaliação da escala SAB pode servir como norteadora no âmbito escolar, se trabalhada com os professores ou cuidadores.

Palavras-chave: Sistema Nervoso; Doenças do Sistema Nervoso; Audição; Percepção Auditiva; Transtornos da Audição.

\section{Abstract}

The use of questionnaires can help to verify the perception of parents / guardians about their children's hearing abilities. To compare the perception of the parents of schoolchildren submitted to acoustically controlled auditory training, in the initial and final moments, through a questionnaire called SAB Scale. Method: 18 children (66.6\% boys) aged 6 to 13 years (mean of 10.4 years) underwent auditory training, 15 sessions of 45 minutes, after being diagnosed with Central Auditory Processing Disorder. This questionnaire contains 12 questions about auditory behavior which total score ranges from 12 (lowest score) to 60 points (highest score). These questions were grouped by domain: attention, hearing, learning, listening comprehension. Results: In the perception of parents / guardians through SAB scale, the greatest improvement was perceived in the field of hearing, followed by attention, learning and listening comprehension behaviors. Conclusion: The parents perceived evolution of the auditory behavior of their children with satisfactory improvement in the areas of hearing and attention. The understanding and learning domains did not reach the same improvement as before. The evaluation of the SAB scale serves as a guide in the school context if it is worked with teachers or caregivers.

Keywords: Nervous System; Nervous System Diseases; Hearing; Auditory Perception; Hearing Disorders.

\section{Resumen}

El uso de cuestionarios puede ayudar a verificar la percepción de padres / tutores sobre la capacidad auditiva de sus hijos Objetivo. Comparar la percepción de los padres de escolares sometidos al entrenamiento auditivo acústicamente controlado, en los momentos de inicio y de final, mediante el uso del cuestionario Scale Auditory Behavior,denominado "escala SAB". Método: 18 niños (66,6\% varones), después de diagnosticados con Trastorno de Procesamiento Auditivo Central (TPAC),de edades comprendidas entre los 6 y 13 años (promedio 10,4 años) fueron sometidos al entrenamiento auditivo que duró 15 sesiones de 45 minutos cada. Todos los participantes tuvieron una mejoría en la evaluación de la conducta posterior a la intervención. Los responsables fueron sometidos a la escala SAP antes y 
después del entrenamiento auditivo. El cuestionario contiene 12 preguntas sobre el comportamiento auditivo, cuya puntuación total oscila entre 12 (puntuación más baja) a 60 puntos (máxima puntuación). Las preguntas fueron agrupadas por dominios: atención, audición, aprendizaje, comprensión auditiva. Resultados: En la percepción de los padres / tutores a través de la escala SAB la principal mejora se observó en el dominio de la audición, seguida de los comportamientosauditivos de atención,aprendizaje y comprensión auditiva. Conclusiones: Los padres de los niños sometidos a la intervención se dieron cuenta de la progresión de la conducta auditiva de sus hijos con una mejoría satisfactoria predominantemente en los dominiosaudición y atención. Los dominios de comprensión aprendizaje no alcanzaron la misma mejora de las anteriores. La evaluación de la escala SAB puede servircomo una guía en la escuela si trabajada con los maestros o cuidadores.

Palabras claves: Sistema Nervioso; Enfermedades del Sistema Nervioso; Audición; Percepción Auditiva; Trastornos de la Audición.

\section{Introdução}

A audição é uma das modalidades sensoriais que permite ao sujeito o desenvolvimento de competências fundamentais, como interagir, comunicar-se e aprender. A partir da apreciação do som, é possível desempenhar diversos comportamentos complexos e, ao mesmo tempo, inerentes à natureza humana. ${ }^{1-3}$

A participação do sistema auditivo nesses comportamentos depende dos processos realizados em níveis sensoriais, cognitivos e linguísticos a partir de habilidades auditivas adquiridas e desenvolvidas da primeira infância à adolescência. ${ }^{4,5}$

O processamento auditivo central diz respeito a esses fenômenos e pode ser definido pelo conjunto de habilidades necessárias à interpretação dos sons, tais como detecção, discriminação, reconhecimento e memória. Assim, defasagens nessas habilidades podem interferir nos comportamentos cotidianos do sujeito. ${ }^{6}$ Testes da Avaliação do Processamento Auditivo podem indicar déficits em certas habilidades auditivas. ${ }^{7,8}$

Estudos mostram que o Treinamento Auditivo Acusticamente Controlado (TAAC) aprimora o aprendizado perceptual, por meio de vários controles acústicos, desde o ambiente de estimulação até o registro das respostas e, predominantemente, dos estímulos, incluindo as variações de silêncio entre os estímulos e a variação da frequência e duração sonora. ${ }^{9}$

Os resultados positivos do TAAC podem ser observados no comportamento auditivo do indivíduo, repercutindo no âmbito comunicativo e acadêmico. ${ }^{10}$ Instrumentos como a Scale of Auditory Behavior, denominado "escala SAB" - um questionário que avalia o comportamento auditivo - podem determinar o impacto desse déficit na vida de um indivíduo em situações diárias de comunicação e aprendizagem. ${ }^{11}$

Neste estudo-piloto, as 12 (doze) questões da escala foram divididas e reagrupadas para a identificação de comportamentos específicos relacionados aos domínios de Atenção, Compreensão, Audição e Aprendizado.

Este trabalho teve por objetivo comparar a percepção dos pais de escolares submetidos ao TAAC, nos momentos pré e pós, segundo diferentes domínios do comportamento auditivo elencados a partir da escala SAB.

\section{Método}

O presente estudo foi realizado após a aprovação do Comitê de Ética em Pesquisa sob o número 319.303.

Contou com os dados obtidos nos prontuários de 18 crianças ( $66,6 \%$ meninos), com faixa etária entre 6 (seis) a 13 (treze) anos (média de 10,4 anos), diagnosticadas com Transtorno do Processamento Auditivo Central. O estudo contemplou, ainda, as respostas do questionário Scale of Auditory Behaviors $(\mathrm{SAB})$, respondidas pelos respectivos pais nos momentos pré-treinamento e pós-treinamento auditivo em um serviço público de atendimento ambulatorial. Todos os indivíduos apresentaram melhora na avaliação do processamento auditivo após TAAC.

A abordagem de intervenção foi a denominada Treinamento Auditivo Acusticamente Controlado (TAAC). Foi realizada de forma individual, em sessões com duração de 45 minutos uma vez por 
semana. Para tanto, o indivíduo foi posicionado no interior de uma cabina acústica para controlar o nível de ruído do ambiente de estimulação. Os níveis sonoros dos estímulos foram controlados acusticamente, bem como os registros das respostas. A abordagem foi realizada conforme o proposto em vários estudos. ${ }^{12-16}$ Os pais foram submetidos a um questionário, denominado Escala $\mathrm{SAB}$, previamente ao TAAC, o "momento Pré" e no final do TAAC, denominado "momento Pós". O questionário contém 12 (doze) questões sobre o comportamento auditivo diário. Para cada questão, há cinco possibilidades de respostas que variam em pontuação de um a cinco, sendo: frequente (1); quase sempre (2); algumas vezes (3); esporádico (4); e nunca (5). A pontuação de comportamento auditivo satisfatório corresponde a 5 (cinco) pontos, enquanto o insatisfatório, a 1 (um) ponto, em cada uma das questões. Assim, a pontuação total pode variar de 12 a 60 pontos.

A análise dos dados da Escala SAB foi realizada por meio de um agrupamento das questões por domínios. Essa nova configuração da Escala é realizada com o auxílio de uma psicóloga, membro do grupo de pesquisa, e propõe 4 (quatro) domínios estabelecidos, considerando a uma abordagem da psicologia comportamental e da audiologia, a saber: Compreensão, Aprendizagem, Atenção e Audição.

Nesse contexto, o domínio Compreensão refere-se à capacidade de entender instruções dadas, sem que seja necessário repeti-las, ou seja, uma prontidão maior para compreender as informações. Assim as, questões (3) "dificuldade de seguir instruções orais" e (7) "pede para repetir as coisas" foram selecionadas para este domínio.

Já para o domínio Aprendizagem - relacionado à capacidade de absorção de conhecimentos ${ }^{17}$ tais como leitura, escrita e verbalização, entre outros comuns no ambiente acadêmico -, foram selecionadas as questões (6) "pobre habilidade de leitura", (9) "dificuldades acadêmicas ou de aprendizado" e (12) "desorganizado".

Para o domínio Atenção, considerando a definição em um contexto mais amplo, selecionamos as seguintes questões: (8) "facilmente distraído", (10) "período de atenção curto" e (11) "sonha durante o dia, desatento". Esses aspectos atencionais são estudados por diferentes áreas do conhecimento, sendo considerados como importantes constructos para a compreensão dos processos perceptivos e cognitivos. ${ }^{13,18}$
A atenção, em conceituação mais ampla ${ }^{19}$, administra as informações percebidas, as memorizadas e as decorrentes de outros processos cognitivos e, se origina da capacidade do cérebro em priorizar um estimulo sobreposto a vários outros.

Para o domínio Audição, considerou-se o conceito ampliado, desde a detecção até o reconhecimento de fala em diferentes situações de baixa redundância ${ }^{20,21}$, sendo selecionadas as seguintes questões: (1) "dificuldade em escutar ou entender em ambiente ruidoso"; (2) "não entender bem quando alguém fala rápido ou "abafado"; (4) "dificuldade na identificação e discriminação dos sons de fala"; e (5) "inconsistência de respostas para informações auditivas".

Sendo assim, os quatro domínios estabelecidos foram: Compreensão (2 a 10 pontos); Aprendizagem (3 a 15 pontos); Atenção ( 3 a 15 pontos); e Audição (4 a 20 pontos).

A análise dos dados foi realizada por meio da contabilidade das ocorrências de respostas por questão segundo a classificação e seu peso correspondente. Denominaram-se duas nomenclaturas: "SAB_total" para o total de pontuação das 12 questões; e "SAB_domínio" para o total de questões por domínio: Atenção, Compreensão, Aprendizado e Audição.

Os dados obtidos dos escores pré e pós foram transformados em porcentagem de dificuldade. A seguir, calculou-se a diferença entre o escore esperado, isto é, 5 pontos em uma questão ou 60 pontos no total e o escore observado, denominando-se esse valor como "porcentagem de dificuldade_SAB" ("SAB_dificuldade"). A diferença entre os valores de dificuldade_SAB pré e pós foi denominado de "evolução do comportamento auditivo na visão dos pais (SAB_evolução).

Foi realizada a estatística descritiva - valor da média, do desvio-padrão, da mediana, valor mínimo e valor máximo - das pontuações $\mathrm{SAB}$ total; SAB_dominio e SAB_dificuldade; e SAB_evolução. A análise estatística inferencial foi realizada por meio dos testes não paramétricos. Para a análise da contribuição de cada domínio na contabilização do escore final, tanto no momento pré como no momento pós TAAC, foram utilizados os testes de Wilcoxon e Kruskal Wallis. O nível de significância adotado foi de $5 \%$. 


\section{Resultados}

A ocorrência individual da pontuação $\mathrm{SAB}$ total, SAB_dificuldade e SAB_evolução nos momentos pré e pós é apresentada na Tabela 1 .

Os dados da estatística descritiva para o SAB total nos momentos pré e pós foram mostrados na Tabela 2 e SAB_dificuldade e SAB_evolução na Tabela 3.

Tabela 1. Ocorrência individual da pontuação SAB_total, SAB_dificuldade e SAB_evolução.

\begin{tabular}{cccc}
\hline & $\begin{array}{c}\text { SAB_total } \\
\text { (SAB_dificuldade em \%) }\end{array}$ & $\begin{array}{c}\text { SAB_diferença (Pós-Pré) } \\
\text { (SAB_evolução em \%) }\end{array}$ \\
\hline $\mathbf{N}$ & Pré & Pós & $20(33.33)$ \\
1 & $20(66.67)$ & $40(33.33)$ & $8(13.33)$ \\
2 & $29(51.67)$ & $37(38.33)$ & $31(51.67)$ \\
3 & $15(75.00)$ & $46(23.33)$ & $28(46.67)$ \\
4 & $16(73.33)$ & $44(26.67)$ & $27(45.00)$ \\
5 & $19(68.33)$ & $46(23.33)$ & $28(46.67)$ \\
6 & $14(76.67)$ & $42(30.00)$ & $15(25.00)$ \\
7 & $24(60.00)$ & $39(35.00)$ & $11(18.33)$ \\
8 & $20(66.67)$ & $31(48.33)$ & $12(20.00)$ \\
9 & $42(30.00)$ & $54(10.00)$ & $8(25.00)$ \\
10 & $25(58.33)$ & $40(33.33)$ & $9(13.33)$ \\
11 & $18(70.00)$ & $26(56.67)$ & $16(26.67)$ \\
12 & $33(45.00)$ & $42(30.00)$ & $7(11.67)$ \\
13 & $24(60.00)$ & $40(33.33)$ & $12(20.00)$ \\
14 & $40(33.33)$ & $47(21.67)$ & $14(23.33)$ \\
15 & $27(55.00)$ & $39(35.00)$ & $27(45.00)$ \\
16 & $16(73.33)$ & $30(50.00)$ & $26(43.33)$ \\
17 & $17(71.67)$ & $44(26.67)$ & \\
18 & $23(61.67)$ & $49(18.33)$ & \\
\hline
\end{tabular}

Legenda: $\mathrm{N}=$ número da observação; $\mathrm{SAB}=$ Scale Auditory Behavior

Tabela 2. Estatística descritiva SAB_total nos momentos pré e pós.

\begin{tabular}{ccccc}
\hline & Pré & Pós & $\begin{array}{c}\text { Diferença de } \\
\text { pontuação }\end{array}$ & $\begin{array}{c}\text { Teste de Wilcoxon } \\
\text { pré x pós }\end{array}$ \\
\hline Mínimo & 14 & 26 & $7(11.67)$ & \\
Máximo & 42 & 54 & $31(51.67)$ & \\
Média & 23,44 & 40,88 & $17.444(29.074)$ & p valor $<0,0001 *$ \\
Variância & 67,20 & 47,75 & $67.908(188.63)$ & \\
Desvio-Padrão & 8,19 & 6,91 & $8.241(13.734)$ & \\
Mediana & 21,5 & 41,0 & $15(25.0)$ & \\
\hline
\end{tabular}

Legenda: *estatisticamente significante; $\mathrm{SAB}=$ Scale Auditory Behavior

Tabela 3. Estatística descritiva SAB_dificuldade nos momentos pré e pós e SAB_evolução.

\begin{tabular}{cccc}
\hline & \multicolumn{2}{c}{ SAB_dificuldade } & \multirow{2}{*}{ SAB_evolução } \\
\cline { 2 - 3 } & pré & pós & 11,66 \\
Mínimo & 30,00 & 10,00 & 51,66 \\
Máximo & 76,66 & 56,66 & 29,07 \\
Média & 60,92 & 31,85 & 188,63 \\
Variância & 186,67 & 132,643 & 13,73 \\
Desvio Padrão & 13,66 & 11,51 & 25,00 \\
Mediana & 64,16 & 31,66 & \\
\hline
\end{tabular}

Legenda: $\mathrm{SAB}=$ Scale Auditory Behavior 
Na Tabela 4, observa-se a SAB_dificuldade por domínio, bem como o valor calculado para a comparação da SAB_dificuldade em porcentagem por domínio entre os momentos pré e pós TAAC.

Considerando os domínios de SAB individualmente, foi analisada a contribuição desses grupos simultaneamente pelo teste de Kruskal Wallis, com o objetivo de identificar se há diferença entre os grupos e entre os domínios nos momentos pré, com valor de $\mathrm{p}(0,1143)$, e no momento pós, com valor de $\mathrm{p}(0,204)$. Assim, os domínios, tanto no momento pré-treinamento como no momento pós-treinamento apresentaram contribuição na composição do escore final de igual.

Neste estudo, nas respostas do questionário, foram verificadas pioras na percepção dos pais em algumas questões no momento pós-TAAC. As ocorrências dessas pioras foram assinaladas individualmente na Figura 1.

Tabela 4. Estatística descritiva da pontuação SAB_dificuldade _\% por domínio da escala SAB e o p-valor calculado para comparação entre os momentos pré e pós.

\begin{tabular}{|c|c|c|c|c|c|c|}
\hline & Média & Mediana & $\begin{array}{l}\text { Desvio- } \\
\text { Padrão }\end{array}$ & Q1 & Q3 & $\begin{array}{l}\text { Valor de } p \\
\text { Pré } x \text { pós }\end{array}$ \\
\hline Pré - Total & 60,92 & 64,16 & 13,66 & 55.00 & 71.66 & \multirow{2}{*}{$0.00020 *$} \\
\hline Pós - Total & 31,85 & 31,66 & 11,51 & 23.33 & 35.00 & \\
\hline Pré - Audição & 56.38 & 55.00 & 13.26 & 50.00 & 65.00 & \multirow{2}{*}{$0.00019 *$} \\
\hline Pós - Audição & 27.22 & 25.00 & 13.95 & 15.00 & 35.00 & \\
\hline $\begin{array}{c}\text { Pré - } \\
\text { Compreensão }\end{array}$ & 60.00 & 60.00 & 19.70 & 50.00 & 80.00 & \multirow{2}{*}{$0.00145^{*}$} \\
\hline $\begin{array}{c}\text { Pós - } \\
\text { Compreensão }\end{array}$ & 31.11 & 30.00 & 14.09 & 20.00 & 40.00 & \\
\hline Pré - Atenção & 61.85 & 66.66 & 18.82 & 53.33 & 80.00 & \multirow{2}{*}{$0.00059 *$} \\
\hline Pós - Atenção & 32.59 & 33.33 & 15.98 & 26.66 & 40.000 & \\
\hline $\begin{array}{c}\text { Pré - } \\
\text { Aprendizado }\end{array}$ & 66.67 & 76.67 & 19.80 & 60.00 & 80.000 & \multirow{2}{*}{$0.00027 *$} \\
\hline $\begin{array}{c}\text { Pós - } \\
\text { Aprendizado }\end{array}$ & 37,78 & 33,33 & 15,842 & 33,33 & 46.66 & \\
\hline
\end{tabular}

Legenda: $\mathrm{SAB}=$ Scale Auditory Behavior ; * estatisticamente significante; $\mathrm{p}=$ teste de Wilcoxon

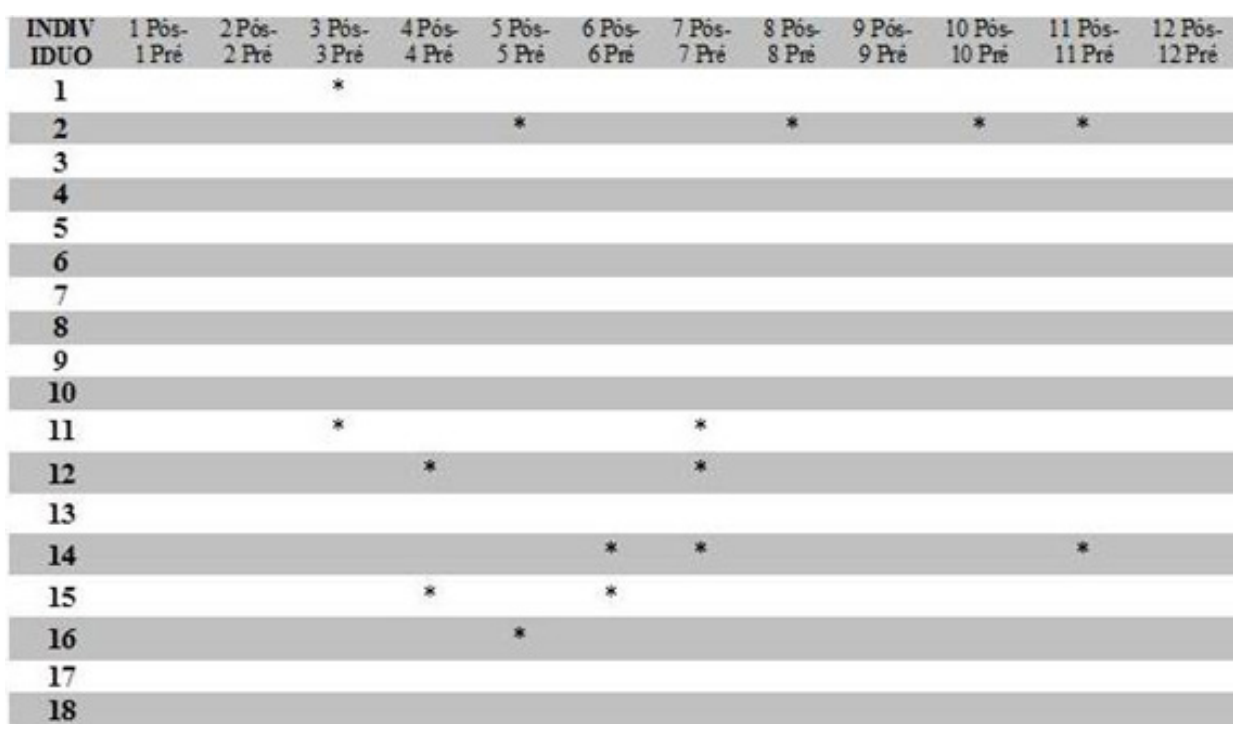

Legenda: $*=$ indicam quando determinado comportamento se tornou pior, segundo escala SAB, após o período de tratamento.

Figura 1. Ocorrência de piora no comportamento auditivo na percepção dos pais obtidas por meio da Escala SAB por indivíduo. 


\section{Discussão}

Como se trata de um estudo-piloto, a amostra não é suficiente para determinar o comportamento de uma população. Sugere-se que estudos venham a aumentar a amostra.

Após o TAAC, a evolução do comportamento auditivo na percepção dos pais não só melhorou (Tabela 1, Tabela 2, Tabela 3), mas foi significativa (Tabela 4).

Como apresentado na Tabela 1, na aplicação da escala $\mathrm{SAB}$ pré-treinamento, os 18 participantes da pesquisa apresentaram escore total de avaliação $\mathrm{SAB}$ menor que 46 pontos, sendo que dois tiveram escore menor que 46 pontos e maior que 35 , e os dezesseis restantes tiveram escore menor que 35 pontos. Após o TAAC, todos os pacientes tiveram evolução do comportamento auditivo, que, percentualmente, variou de $11,67 \%$ a 51,67\%. Destes, três (observação 8, 11 e 16) mantiveram-se com escore abaixo de 35 pontos, e o restante com pontuações maiores. Na literatura especializada, um estudo ${ }^{11}$ mostrou que, na escala $\mathrm{SAB}$, pontuações inferiores a 35 indicam elevado risco para alteração de processamento auditivo. Por seu turno, na mesma escala, a partir de 46 pontos, há baixo risco para a mesma alteração. Assim, neste estudo, apenas três $(16,66 \%)$ indivíduos ainda mantiveram elevado risco de alteração de processamento após o TAAC. Portanto, o sucesso dessa intervenção mensurada pela escala SAB ocorreu em $83,33 \%$ da amostra na percepção dos pais.

$\mathrm{Na}$ análise realizada por domínios, foi verificada similaridade de dificuldade em cada um dos momentos, pré e pós-intervenção.

Houve evolução do comportamento auditivo, considerando cada um dos domínios (Tabela 4), com uma melhora significativa (em torno de $30 \%$ ) para cada um dos domínios. As melhoras mais elevadas (Tabela 4) ocorreram nos domínios Audição e Atenção, enquanto os domínios Compreensão e Aprendizado apresentaram melhoras menos ostensivas. Uma das justificativas possíveis para que os domínios Audição e Atenção exibam melhor desempenho em relação aos outros dois domínios se deve ao fato de que o TAAC é um treinamento especifico para o desenvolvimento das habilidades auditivas e atenção auditiva, conforme alguns estudos apontam. ${ }^{6,8,11,12}$

Outros estudos indicam que, por meio de um programa de treinamento auditivo, o sistema auditi- vo central pode ser modificado, revelando a plasticidade $^{22}$ do sistema nervoso central. Contudo, para a efetiva melhora das habilidades de aprendizagem, linguagem, leitura e escrita - que se encontram nos domínios Compreensão e Aprendizagem -, são necessárias, além do TAAC, outras intervenções fonoaudiológicas globais ${ }^{10,13}$.

Outro ponto importante a se considerar diz respeito à avaliação dos pais em relação ao comportamento auditivo de alguns indivíduos, indicando piora em algumas das questões no momento pós-TAAC (Figura 1). Por exemplo, nos indivíduos 11 e 12, a piora ocorreu em questões envolvendo o domínio Compreensão. Nos indivíduos 2 e 11, a piora ocorreu para questões do domínio Atenção. No individuo 14, em Aprendizagem. Diante disso, cabe a indagação: as alternâncias em consistência das respostas, nos domínios Compreensão, Atenção e Aprendizado, podem ser efeito da terapia ou do questionário? Uma das respostas tem como referência os resultados positivos da avaliação do processamento auditivo pós-TAAC, que descarta a possibilidade de a alteração advir do treinamento acusticamente controlado. Não obstante, podemos justificar por alguns aspectos que podem ser de cunho psicológico ou atencional, conforme dupla topicalização abaixo.

Psicológico - quando os pais respondem a algum tipo de questionário, ou até mesmo escala, como foi o caso da $\mathrm{SAB}$, através da qual há perguntas que podem instigar o desconforto por alguma situação vivida, é possível que se utilizem do mecanismo de enfrentamento, que é a negação psíquica do problema ${ }^{23}$. Assim, deixam de perceber alguma dificuldade da criança mencionada na escala. É uma estratégia usada pelos pais ou responsáveis, que muitas vezes, não têm consciência de que estejam agindo desta forma. A tarefa da psique ${ }^{24}$ diz respeito a encontrar estratégias de enfrentar a ansiedade ${ }^{25}$, a qual é provocada por um aumento da tensão ou desprazer, podendo surgir em qualquer situação real ou imaginária que ameace a integridade física ou psíquica, sobretudo quando a situação é forte demais para ser ignorada, dominada ou descarregada. As situações que venham a causar ansiedade incluem, por exemplo a perda de um objeto desejado, além da rejeição ou mesmo do fracasso em obter o alvo pretendido. O que ocorreu neste estudo, na visão dos pais acerca da dificuldade de seus filhos, pode ser o que ocorre geralmente quando os pais tendem a utilizar a negação como uma estratégia 
para justificar transtornos de comportamento que os filhos apresentam. Além disso, costumam adotar uma tentativa de negação para não aceitar de forma consciente algum fato que venha a desestabilizar o ego. Adultos que tendem a não enxergar fatos reais fantasiam os acontecimentos, fogem em vez de encarar os problemas.

Atencional - os pais podem não ter sido sensibilizados para alguns comportamentos de seus filhos. Porém, após o contato com as questões da escala $\mathrm{SAB}$ passaram a perceber comportamentos antes não observados.

$\mathrm{Na}$ literatura especializada, vários trabalhos 26-29 demonstraram a efetividade do TAAC em diferentes populações, e esse aprimoramento se deve a mudanças estruturais, conforme um estudo que demonstra que as conexões neurobiológicas ${ }^{30}$ são a chave para a intervenção em alterações do processamento auditivo. Neste estudo, verificou-se que o aprimoramento do comportamento auditivo pode ser observado pelos pais por meio de um instrumento de fácil aplicação - no caso, a escala $\mathrm{SAB}$-, que também auxilia na sensibilização dos pais para que se atenham ao comportamento auditivo de seus filhos.

Para controlar a variável atencional da percepção dos pais ao responderem as questões da escala $\mathrm{SAB}$, recomenda-se aplicar o questionário duas vezes antes da intervenção TAAC e considerar as respostas da segunda aplicação como referência. Quanto à variável psicológica, pode-se pensar na contribuição de uma abordagem terapêutica junto aos pais para sensibilização e desenvolvimento de um olhar especifico em relação ao comportamento auditivo de seus filhos.

A avaliação da escala SAB pode servir como norteador no âmbito escolar, se trabalhada com os professores ou cuidadores.

\section{Conclusão}

Os pais das crianças submetidas à intervenção perceberam evolução do comportamento auditivo de seus filhos com melhora satisfatória. É possível, através da escala SAB, perceber alterações específicas do comportamento auditivo e classificá-las por domínios, o que possibilita o treinamento direcionado para cada domínio alterado.

\section{Referências bibliográficas}

1. Russo ICP. Editorial II: a relevância da pesquisa científica na audiologia brasileira. Rev. CEFAC [Internet]. 2009 [acesso em: dezembro 2016 ] ; 11( Suppl 1 ). Available from: http:// www.scielo.br/scielo.php?script=sci_arttext\&pid $=$ S151618462009000500002\&lng=en. http://dx.doi.org/10.1590/ S1516-18462009000500002.

2. Etges Camila Lucia, Reis Mariana Citton Padilha dos, Menegotto Isabela Hoffmeister, Sleifer Pricila, Soldera Cristina Loureiro Chaves. Achados na triagem imitanciométrica e de processamento auditivo em escolares. Rev. CEFAC [Internet]. 2012 Dec [cited 2017 Feb 24]; 14(6): 1098-1107. Available from: $\mathrm{http}: / /$ www.scielo.br/scielo.php?script=sci_arttext\&pid=S151618462012000600011\&lng=en. Epub Apr 05, 2012. http:// dx.doi.org/10.1590/S1516-18462012005000028.

3. Pereira, LD, Schochat E. Processamento auditivo central: Manual de avaliação. 1ed São Paulo, SP: Lovise, 1997

4. Terto, SSM, Lemos, SM A. Aspectos temporais auditivos em adolescentes do $6^{\circ}$ ano do ensino fundamental. Rev. CEFAC [online]. 2013, vol.15, n.2 [acesso em: dezembro, 2016], pp.271-286. Disponível em: http://www.scielo.br/scielo. php?script $=$ sci_arttext\&pid=S1516-18462013000200003\&lng $=$ en\&nrm=iso

5. Fridlin SL, Pereira LD, Perez, AP. Relação entre dados coletados na anamnese e distúrbio do processamento auditivo. Rev. CEFAC [online]. 2014, vol.16, n.2 [acesso em: dezembro, 2016], pp.405-412. Disponível em: http://www.scielo.br/scielo. php?script=sci_arttext\&pid=S1516-18462014000200405\&lng $=$ en\&nrm=iso

6. Vargas GC, Ferreira MIDC, Vidor DCGM, Machado MS. Avaliação simplificada e comportamental do processamento auditivo em escolares: estabelecendo relações. Rev. CEFAC [online]. 2014, vol.16, n.4 [acesso em dezembro, 2016], pp.1069-1077. Disponível em : http://www.scielo.br/scielo. php?script=sci_arttext\&pid=S1516-18462014000401069\&ln $\mathrm{g}=$ en\&nrm=iso

7. Gallo J, et al. Avaliação do processamento auditivo em crianças nascidas pré-termo. J. Soc. Bras. Fonoaudiol. [online]. 2011, vol.23, n.2 [acesso em: dezembro, 2016], pp.95-101. Disponível em: http://www.scielo.br/scielo.php?script=sci arttext\&pid=S2179-64912011000200003\&lng=en\&nrm=iso

8. Caumo DTM, Ferreira MDC. Relação entre desvios fonológicos e processamento auditivo. Rev. soc. bras. fonoaudiol. [online]. 2009, vol.14, n.2 [acesso em: novembro, 2016], pp.234-240. Disponível em: http://www.scielo.br/scielo. php?script $=$ sci_arttext\&pid=S1516-80342009000200015\&lng $=$ en\&nrm=iso

9. Cruz ACA, Andrade NA, Gil D. A eficácia do treinamento auditivo formal em adultos com distúrbio do processamento auditivo (central). Rev. CEFAC [online]. 2013, vol.15, n.6 [acesso em: outubro, 2016], pp.1427-1434. Disponível em: http://www.scielo.br/scielo.php?script=sci_arttext\&pid=S1516$18462013000600004 \& \operatorname{lng}=$ en\&nrm=iso.

10. Avila RRA, Murphy CRB, Schochat E. Efeitos do Treinamento Auditivo em Idosos com comprometimento Cognitivo Leve. Psicol. Reflexo. Crit. [conectados]. 2014, vol.27, n.3 [acesso em novembro, 2016], pp.547-555. Disponível em: http://www.scielo.br/scielo.php?script=sci_arttext\&pid=S010279722014000300547\&lng=en\&nrm=iso 
11. Nunes CL, Pereira LD, Carvalho GS. Scale of Auditory Behaviors e testes auditivos comportamentais para avaliação do processamento auditivo em crianças falantes do português europeu. CoDAS [online]. 2013, vol.25, n.3 [acesso em: setembro, 2016], pp.209-215. Disponível em: http://www.scielo.br/scielo. php?script=sci_arttext\&pid=S2317-17822013000300004\&lng $=$ en\&nrm $=$ iso

12. Gil D, Iório MCM. Treinamento auditivo formal em adultos com deficiência auditiva. [Tese Doutorado]. São Paulo: Universidade Federal de São Paulo. Escola Paulista de Medicina; 2006.

13. Ziliotto K, Pereira LD. Random gap detection test in subjects with and without APD. In: 17th American Academy of Audiology - Annual Convention and Exposition; 2005; Washington (DC).p.30

14. Cibian, AP, Pereira LD. Figura-fundo em tarefa dicótica e sua relação com habilidades não treinadas / Figure-background in dichotic task and their relation to skills untrained. CoDAS; 27(5): 419-427, set.-out. 2015.

15. Musiek F, Schochat E. Auditory training and central auditory processing disorders: a case study. Semin Hear. 1998; 19(4): 357-66.

16. Murphy CF, Schochat E. Generalization of temporal order detection skill learning: two experimental studies of children with dyslexia. Braz J Med Biol Res. 2010; 13(4): 359-66.

17. Rosário P, Almeida L.. Leituras construtivistas da aprendizagem. Em: G. Miranda; S. Bahia (Eds.). Psicologia da educação: temas de desenvolvimento, aprendizagem e ensino (141-165). Lisboa: Relógio D’água Editores - 2005

18. Lima RF. Compreendendo os Mecanismos Atencionais. Ciências \& Cognição, [S.1.], v. 6, Nov. 2005. Acesso em: 30 novembro 2016. Disponível em: http://www. cienciasecognicao.org/revista/index.php/cec/article/view/537

19. Rued FJM, Castro NR. Capacidade atencional: há decréscimo com o passar da idade? . Psicol. Cienc. Prof, Brasília, v. 30, n.3 , sept 2010- Disponível em http://www. scielo.br/scielo.php?
20. Boothroyd A. Perception of speech pattern contrasts from auditory presentation of voice fundamental frequency. 1988 Dec; 9(6): 313-21

21. ASHA: Working Group on Auditory Processing Disorders. (Central) Auditory Processing Disorders. Rockville, MD: American Speech Language-Hearing Association; 2005. Report $\mathrm{N}^{\mathrm{o}}$ : TR2005-00043

22 Musiek F, Shinn J, Hare C. Plasticity, auditory training, and auditory processing disorders. Seminars in Hearing. 2002; 23(4): 263-75.

23. Neves IF, Schochat E. Maturação do processamento auditivo em crianças com e sem dificuldades escolares. PróFono R. Atual. Cient. [Internet]. 2005 Dec [acesso em: janeiro, 2017] ; 17( 3 ): 311-320. Disponível em: http:// www.scielo.br/scielo.php?script $=$ sci_arttext $\&$ pid $=$ S0104$56872005000300005 \& \operatorname{lng}=\mathrm{e}$

24. Freud S. Psicologia das massas e análise do eu: 1921. In: Obras psicológicas completas de Sigmund Freud. Rio de Janeiro: Imago; 1996.

25. Silva CAB. A ambiguidade genital na percepção dos pais. Rev. Bras. Saúde Matern. Infant., Recife, 6 (1): 107-113, jan. / mar., 2006.

26. Samelli AG, Meca FFDN. Treinamento auditivo para transtorno do processamento auditivo. Rev CEFAC. 2010; 12(2): 235-41.

27. Schochat E, Carvalho LZ, Megale R. Treinamento auditivo: avaliação da manutenção das habilidades. Pró-Fono R. Atual. Cient. 2002; 14(1): 93-9.

28. Zalcman TE, Schochat E. A eficácia do treinamento auditivo formal em indivíduos com transtorno de processamento auditivo. Rev Soc Bras Fonoaudiol. 2007; 12(4): 310-4.

29. Kozlowskil L, Wiemes GMR, Magni C, Silva ALG. A efetividade do treinamento auditivo na desordem do processamento auditivo central: estudo de caso. Braz J Otorhinolaryngol. 2004; 70(3): 427-32.

30. Chermak GD. Neurobiological connections are key to APD. Hear J. 2004; 57(4): 58-9. 